\title{
32 Health risks from heating food II
}

It is primarily red meat (beef, pork lamb) that heating can turn into the source of several carcinogenic agents. Ranking alongside polycyclic hydrocarbons and heterocyclic aromatic amines are the nitrosamines. Even pathogenic viruses in the meat might be causes of cancer, as the Nobel Prize Laureate Harald zur Hausen suspected. Several studies, including the 2 analyses of the prospective NIH-AARP study ( $\downarrow$ Chapter 3 ) from the USA on over 500,000 persons aged between $50-71$ years, have shown that frequent ingestion of processed and unprocessed meat from female cows increases the risk of esophageal, lung, liver and bowel cancer (Sinha et al. 2009, Cross et al. 2007, 2010, Keszei et al. 2012).

According to the results of a large meta-analysis, daily consumption of as little as $50 \mathrm{~g}$ of processed red meat in the form of sausages, bacon, hamburgers, kebab etc. is associated with an additional increase of around $40 \%$ in the risk for cardiovascular diseases and approximately $19 \%$ in the risk for type 2 diabetes (Micha et al. 2010). Data from the EPIC study prove that the risk of early death is $18 \%$ higher in persons exhibiting this dietary behavior (Rohrmann et al. 2013). Causes attributed to this include the higher salt content and the increased number of nitrosamines produced by the heating process involved in cooking these meats. They potentially also promote the development of Alzheimer's disease. Regarding type 2 diabetes, according to data from very large long-term studies, even unprocessed red meat significantly increases the risk of disease (Pan et al. 2011, 2013).

Major speculation is linked to the question as to whether acrylamide, long known as a neurotoxin,

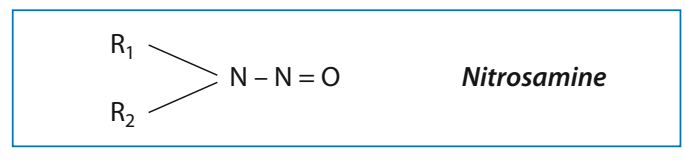

Fig. 32.1

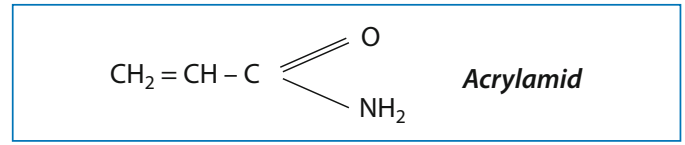

Fig. 32.2

is also mutagenic and can cause cancer as well. Acrylamide and its similarly very harmful, oxidative metabolite glycidamide are formed from the heating process of anhydrous, starch- or sugar-containing foods with the amino acid asparagine.

Acrylamide exposure is particularly high in French fries, potato chips and home fries.

This asparagine occurs in dietary proteins. Having said that, humans have been consuming heated food for thousands of years - which is how long acrylamide has been present in our food. That is why the recommendations for lowering any actual or presumed risk currently only refer to the temperatures applied. For example, baking temperatures should not exceed $190^{\circ} \mathrm{C}$ (or $170^{\circ} \mathrm{C}$ in convection ovens) and frying temperatures should not exceed $160^{\circ} \mathrm{C}$. When the food becomes golden brown within the shortest possible baking or frying time, then this has the utmost priority over browning them too dark. 